\title{
P06-07. A GCLP accredited Clinical Trial Laboratory Network in Africa and India: a collaborative effort between IAVI and in country research organizations

\author{
G Stevens*1, P Chetty ${ }^{1}$, J Stout ${ }^{1}$, T Tarragona1 ${ }^{1}$, T Stiles ${ }^{2}$, S Molapo ${ }^{3}$, C Roodt ${ }^{3}$, \\ L Khumalo $^{3}$, J Cox ${ }^{1}$ and J Gilmour ${ }^{1}$
}

\author{
Address: ${ }^{1}$ Research and Development, International Aids Vaccine Initiative, Johannesburg, South Africa, ${ }^{2}$ Qualogy Ltd, Thrapston, UK and \\ ${ }^{3}$ Contract Laboratory Services, Johannesburg, South Africa \\ * Corresponding author
}

from AIDS Vaccine 2009

Paris, France. 19-22 October 2009

Published: 22 October 2009

Retrovirology 2009, 6(Suppl 3):P96 doi:10.1186/1742-4690-6-S3-P96

This abstract is available from: http://www.retrovirology.com/content/6/S3/P96

(C) 2009 Stevens et al; licensee BioMed Central Ltd.

\section{Background}

IAVI is currently undertaking epidemiology studies and phase I/II clinical trials of prophylactic HIV vaccines in India and Africa. Accredited clinical trial laboratories, established as a collaborative effort between in country research organizations and IAVI now exist as a well recognized laboratory network. Good Clinical Laboratory Practice (GCLP) implementation and laboratory standardization is the key to success.

\section{Methods}

\section{Laboratory Standardisation}

The concept of a central Core Laboratory as a foundation for standardization across clinical trial laboratory testing sites has been successfully implemented for both immunogenicity and safety testing. The IAVI Human Immunology Laboratory (HIL) was established as a unique partnership with Imperial College and Chelsea Westminster Healthcare NHS Trust, in London, England. The Clinical Safety Testing Support Laboratory is contracted to Contract Laboratory Services (CLS), Johannesburg, South Africa. Both laboratories provide ongoing support for the following: 1) Site assessment and development; 2) Equipment/reagent supply; 3 ) Validation of assays, standardization of Equipment/Reagents/Consumables; 4) Training; 5) SOP development; 6) GCLP compliance.

\section{GCLP}

GCLP guidelines define the standards expected from regulatory agencies for laboratory practice in clinical trials. IAVI has led the field by introducing a system to facilitate and ensure GCLP implementation and compliance.

\section{Results}

Through this partnership the IAVI Laboratory Support Program has ensured that 9 Clinical Research Laboratories; Pune and Chennai, India; Nairobi and Kilifi, Kenya, Entebbe and Masaka, Uganda, Kigali, Rwanda and Johannesburg and Cape Town, South Africa have received full GCLP accreditation since implementation of the programme in 2004. Four other African laboratories have GCLP accreditation in progress.

\section{Conclusion}

The process of GCLP accreditation builds long lasting capacity for clinical trial support in countries where HIV, $\mathrm{TB}$, Malaria and other vaccines are being tested and also supports the infrastructure for state-of-the art infectious disease research and intervention studies. Accreditation Status of the Collaborating Clinical Research 